\section{An Olfactory Response of Immature Adults of the Desert Locust}

NorRIs has shown ${ }^{1}$ that a stimulus, probably chemical, produced by mature, yellow males of Schistocerca gregaria (Forsk.) accelerates the maturation of immature males and females. In the present work, a volatile substance, which is produced all over the body, has been detected in yellow males. It is not yet possible to say whether the stimulus demonstrated by Norris and the volatile substance are identical, but they have certain properties in common.

Extracts of this latter substance, or parts of a yellow male, held in front of the antennæ of an immature adult of either sex release a special reaction. The antennre begin to move in a non-directional manner, and this is followed by strong agitation of the two pairs of palpi. Then the hind femora begin to vibrate rapidly, the lateral component of the movement being relatively larger than the anteroposterior movement. The amplitude and duration seem to vary with the state of excitement, which depends upon the quantity and possibly the quality of the releasing stimulus. The hind femora while in motion do not touch the elytra, and tests with sonic and ultrasonic microphones have shown that the vibration reaction contains no acoustical com. ponent.

Amputation and masking experiments suggest that the olfactory receptors concerned are in the antennæ. The palpi have been found to be unimportant for the reception of the stimulus.

The substance is soluble in oil and remains active in mature males for 3-4 days after death. Only yellow, fully mature males produce sufficient of it to release the vibration reaction. Young adults show the olfactory response 4-8 days after emergence and continue to do so until they are fully mature. The reaction can also be released independently of olfactory stimulation by tactile, visual or acoustical stimuli. Further work on this chemical stimulus is in progress, and a detailed account will be published elsewhere.

These investigations were supported by a grant from the Anti-Locust Research Centre.

Imperial College Field Station,

W. LOHER Silwood Park

Sunninghill, Berkshire. Jan. 31.

1 Norris, M. J., "Sexual Maturation in the Desert Locust (Schistocerca gregaria Forskal)", Anti-Loenst Bull., 18 (1954).

\section{Facilitated Diffusion and Exchange in the Absorption of Glucose by the Locust, Schistocerca gregaria (Forsk.)}

GLUCOSE absorption in the locust, Schistocerca gregaria (Forsk.), is largely confined to the mid-gut region of the alimentary canal, the uptake proceeding most rapidly from the mid-gut crea ${ }^{2}$. The rate of disappearance of glucose labelled with carbon-14 from the lumen of the mid-gut was found to be related to its rate of conversion to a disaccharide, trehalose, which accumulated in the hæmolymph. When the isolated alimentary canal was suspended in a relatively large volume of circulating poisoned saline, the disappearance of glucose was similar to that in the intact animal. On the basis of these observa.

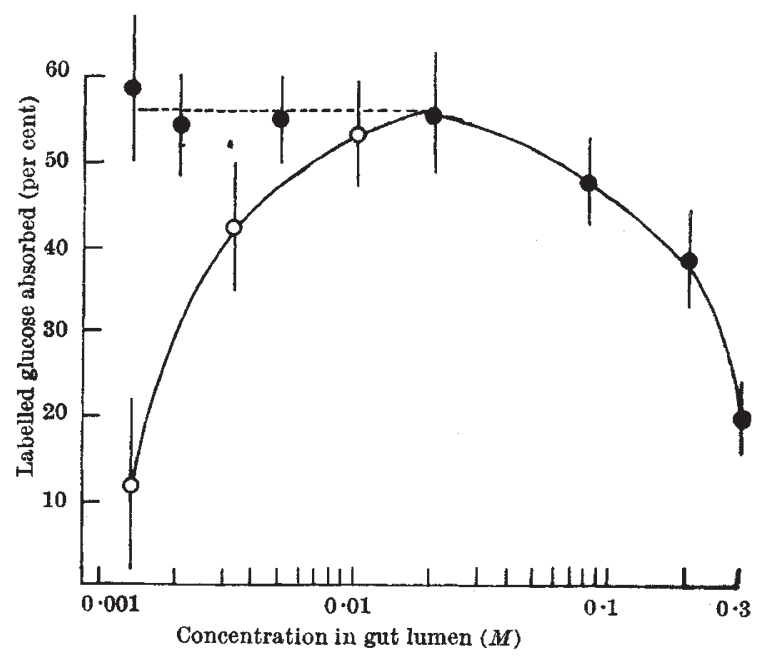

Fig. 1. The effect of concentration on the movements of glucose labelled with carbon-14 between the mid-gut cres and the hamolymph during an experimental period of $15 \cdot 0 \mathrm{~min}$. The open circles represent the percentage absorption of labelled glucose when the initial radioactivity of the glucose in the hromolymph in equilibrium with trehalose, approximated to that in the gut lumen; the closed circles represent the uptake when there was no initial radioactivity in the hæmolymph. The vertical lines indicate the extent of the standard deviation

tions it was suggested that the absorption of glucose might be achieved by diffusion across the gut wall, this process being facilitated by the rapid conversion to trehalose, which would tend to maintain a steep concentration gradient across the gut wall'. This can be described as facilitated diffusion, in the sense that this term is used by Danielli2.

It is known that there is a small amount of glucose in equilibrium with the trehalose in the hæmolymph of this insect ${ }^{3}$. In the present investigation the concentrations were found to be $24 \cdot 1 \pm 12 \cdot 9 \mathrm{mgm}$. $100 \mathrm{ml}$. glucose and $694.5 \pm 99 \cdot 3 \mathrm{mgm} . / 100 \mathrm{ml}$. trehalose. Thus when the glucose in the gut lumen is low, the concentration would be similar to that in the hæmolymph. To investigate the movements of glucose between the gut and the hæmolymph at low concentrations the uptake of labelled glucose

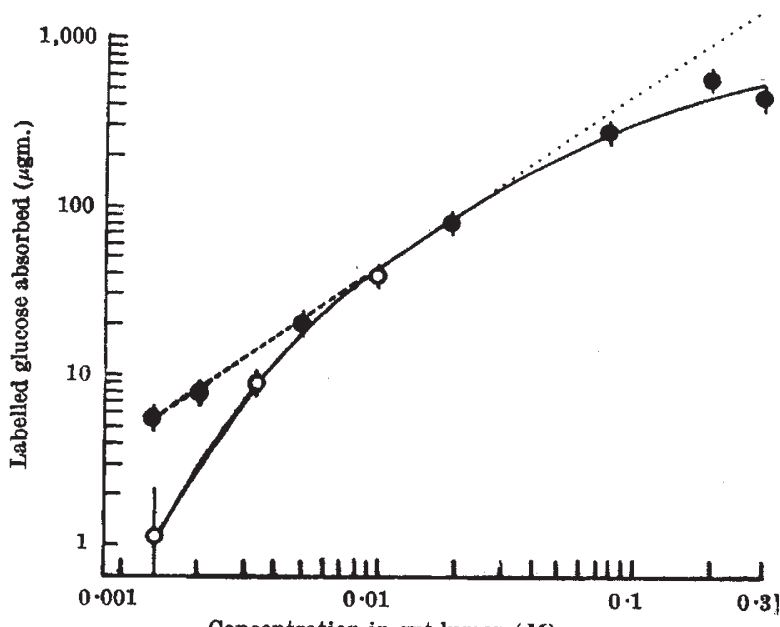

Fig. 2. A logarithmic plot illustrating the uptake of labelled glucose from the mid-gut caeca, in absolute units, calculated from the results illustrated in Fig. 1. The continuous line represents the net glucose absorption, while the broken one includes an estimate of the uptake of labelled glucose resulting from an 